ARTICLE

Received 21 Feb 2014 | Accepted 20 Jun 2014 | Published 21 Jul $2014 \quad$ DOl: 10.1038/ncomms5458

\title{
Rediscovering black phosphorus as an anisotropic layered material for optoelectronics and electronics
}

Fengnian $\mathrm{Xia}^{1, \star}$, Han Wang ${ }^{2, \star} \&$ Yichen $\mathrm{Jia}^{1}$

Graphene and transition metal dichalcogenides (TMDCs) are the two major types of layered materials under intensive investigation. However, the zero-bandgap nature of graphene and the relatively low mobility in TMDCs limit their applications. Here we reintroduce black phosphorus (BP), the most stable allotrope of phosphorus with strong intrinsic in-plane anisotropy, to the layered-material family. For $15-\mathrm{nm}$-thick BP, we measure a Hall mobility of 1,000 and $600 \mathrm{~cm}^{2} \mathrm{~V}^{-1} \mathrm{~s}^{-1}$ for holes along the light $(x)$ and heavy $(y)$ effective mass directions at $120 \mathrm{~K}$. BP thin films also exhibit large and anisotropic in-plane optical conductivity from 2 to $5 \mu \mathrm{m}$. Field-effect transistors using $5 \mathrm{~nm}$ BP along $x$ direction exhibit an on-off current ratio exceeding $10^{5}$, a field-effect mobility of $205 \mathrm{~cm}^{2} \mathrm{~V}^{-1} \mathrm{~s}^{-1}$, and good current saturation characteristics all at room temperature. BP shows great potential for thin-film electronics, infrared optoelectronics and novel devices in which anisotropic properties are desirable.

\footnotetext{
${ }^{1}$ Department of Electrical Engineering, Yale University, New Haven, Connecticut 06511, USA. ${ }^{2}$ IBM Thomas J. Watson Research Center, Yorktown Heights, New York 10598, USA. * These authors contributed equally to this work. Correspondence and requests for materials should be addressed to F.X. (email: fengnian.xia@yale.edu) or to H.W. (email: wanghan@us.ibm.com).
} 
C urrent research in layered materials primarily focuses on graphen $e^{1-10}$, insulating hexagonal boron nitride ${ }^{11-13}$, and members from the transition metal dichalcogenides (TMDCs) $^{14-21}$ family, such as molybdenum disulphide $\left(\mathrm{MoS}_{2}\right)$ and tungsten diselenide ( $\mathrm{WSe}_{2}$ ). Graphene transistors suffer from a low on-off current ratio because of its zero-bandgap nature. Although transistors made from few-layer TMDCs, such as $\mathrm{MoS}_{2}$ and $\mathrm{WSe}_{2}$, show very high on-off current ratios and excellent current saturation characteristics, the carrier mobility in these TMDCs is much lower than in graphene and fluctuates significantly from less than 10 to around $200 \mathrm{~cm}^{2} \mathrm{~V}^{-1} \mathrm{~s}^{-1}$ (refs 21-23), restricting their application in both electronics and optoelectronics. Most of the TMDCs currently being studied have band gaps larger than $1 \mathrm{eV}$, rendering them unsuitable for near-infrared optical telecommunications and mid-infrared applications. Furthermore, in these materials, the photonic, electronic and mechanical properties within the plane of the layers are largely isotropic and do not exhibit a significant directional dependence.

In this work, we reintroduce black phosphorus (BP) to the layered-material family as an anisotropic material for optoelectronic and electronic applications. Narrow gap BP thin film $(0.3 \mathrm{eV}$ in bulk) can fill the space between zero-gap graphene and large-gap TMDCs, making it an ideal material for near and midinfrared optoelectronics. BP thin films with a thickness of $15 \mathrm{~nm}$ show high mobility above $650 \mathrm{~cm}^{2} \mathrm{~V}^{-1} \mathrm{~s}^{-1}$ at room temperature and above $1,000 \mathrm{~cm}^{2} \mathrm{~V}^{-1} \mathrm{~s}^{-1}$ at $120 \mathrm{~K}$ along the light effective mass $(x)$ direction, implying its promising future for high frequency, thin-film electronics. Furthermore, the anisotropic nature within the plane of the layers may allow for the realization of novel thin-film infrared polarizers, infrared polarization sensors, new types of plasmonic devices ${ }^{24}$, phonon transport engineering that can take advantage of the directional dependence of its phonon dispersion and the utilization of the lower effectivecarrier-mass along $x$ direction to build high-performance transistors ${ }^{25-27}$.

\section{Results}

Characterization of BP thin-film layer number. Similar to graphite, bulk BP is an elemental layered material. Bulk BP crystal has been studied extensively decades ago and it is the most stable allotrope of phosphorus ${ }^{28-32}$ because of its unique orthorhombic crystal structure as shown in Fig. 1a. As carbon atoms in graphene, each phosphorus atom is connected to three adjacent phosphorus atoms in BP to form a stable, linked ring structure, with each ring consisting of six phosphorus atoms. However, the puckered BP structure has reduced symmetry compared with graphite, resulting in its unique angle-dependent in-plane conductivities ${ }^{33-36}$. The adjacent layer spacing is around $0.53 \mathrm{~nm}$ and the lattice constant in this orthorhombic system along the $z$ direction is $1.05 \mathrm{~nm}$. Moreover, as in TMDCs, the bandgap in BP is expected to be strongly dependent on the layer number ${ }^{37}$ because of the layer-layer coupling. Single or few-layer $\mathrm{BP}$ can have very different bandstructures compared with its thin film or bulk form. Recent theoretical investigations reveal that for single-layer BP, the single particle band gap can be as large as $2 \mathrm{eV}^{38,39}$. If electron-hole interaction is taken into account, the bandgap can be significantly smaller because of large exciton-binding energy. Furthermore, single and few-layer BP also exhibit strong in-plane anisotropy as in bulk BP. As a result, future exploration on atomically thin BP may lead to new two-dimensional (2D) materials very different from graphene and transition metal dichalcogenides. Recently, the dependence of its band structure on strain was also investigated theoretically ${ }^{40}$.

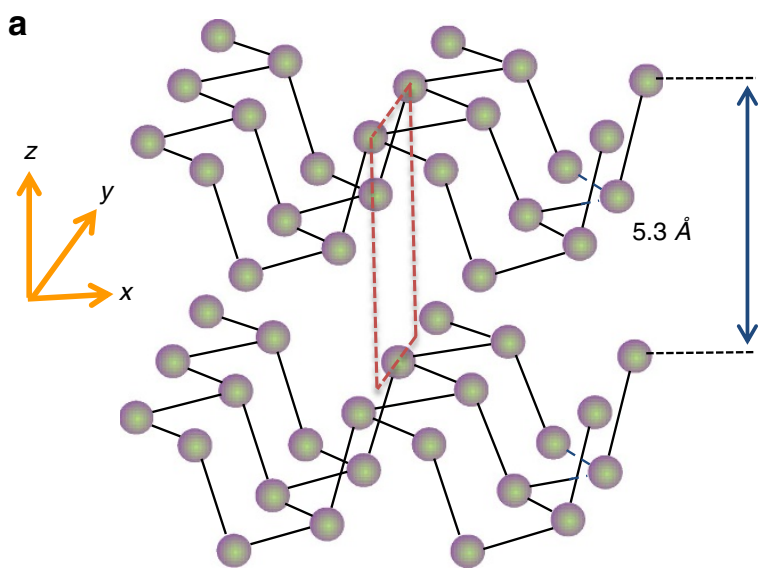

b

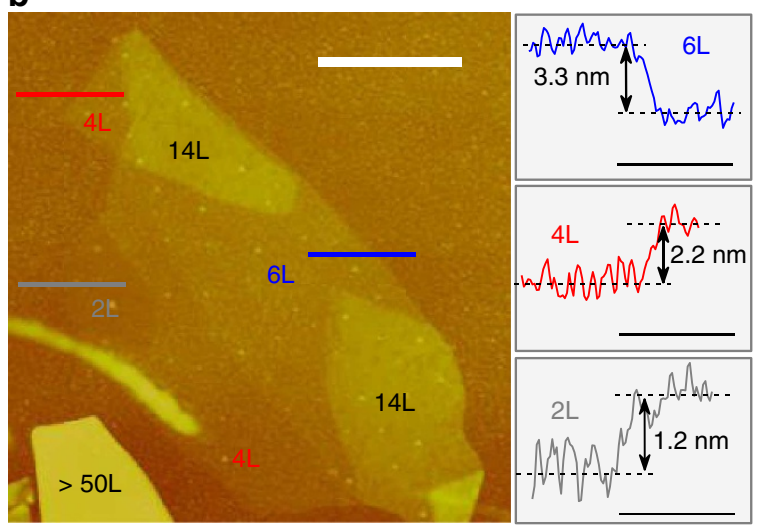

Figure 1 | Characterization of the thickness of black phosphorus.

(a) Layered crystal structure of black phosphorus, showing the relative positioning of two adjacent puckered sheets with linked phosphorus atoms. The BP layer-to-layer spacing is around $5.3 \AA$. (b) Left: an AFM image of black phosphorus flakes. Estimated layer numbers are indicated. Scale bar (thick white): $2 \mu \mathrm{m}$. Right: line scans performed along the coloured lines shown in the left. Layer numbers from 2 to $>50$ are identified based on their thickness. Scale bar (grey): $1 \mu \mathrm{m}$.

In our experiments, multilayer $\mathrm{BP}$ was first exfoliated using the standard mechanical cleavage method from a bulk BP crystal and then deposited on a silicon substrate with 300-nm silicon dioxide. A typical atomic force microscope (AFM) image of thin-film BP is shown in Fig. 1b. Using a layer-to-layer spacing of $0.53 \mathrm{~nm}^{36}$, we clearly identified BP with thicknesses down to around $1.2 \mathrm{~nm}$, corresponding to two atomic layers of BP. However, these fewmicrometre scale bilayer BP flakes are usually too small for optical and electronic experiments. In this work, we performed optical and electrical measurements on BP layers with a thickness varying from 2 to $>30$ nanometres (4-60 layers).

Polarization-resolved infrared spectroscopy. For BP films thicker than $8 \mathrm{~nm}$, flakes with a lateral size in the tens of micrometre scale can be isolated, allowing for the determination of crystalline directions using infrared spectroscopy ${ }^{41,42}$. The lower right inset of Fig. 2a shows an optical image of a BP flake with a thickness of around $30 \mathrm{~nm}$. We measured the polarizationresolved relative extinction spectrum $\left(1-T / T_{0}\right)$ in infrared, where $T$ and $T_{0}$ are the optical transmission through the $\mathrm{BP}$ and the substrate and the substrate only, respectively. Figure 2a denotes $1-T / T_{0}$ measured on $\mathrm{BP}$ with the incident light from the $z$ direction at six different linear polarization angles separated by 

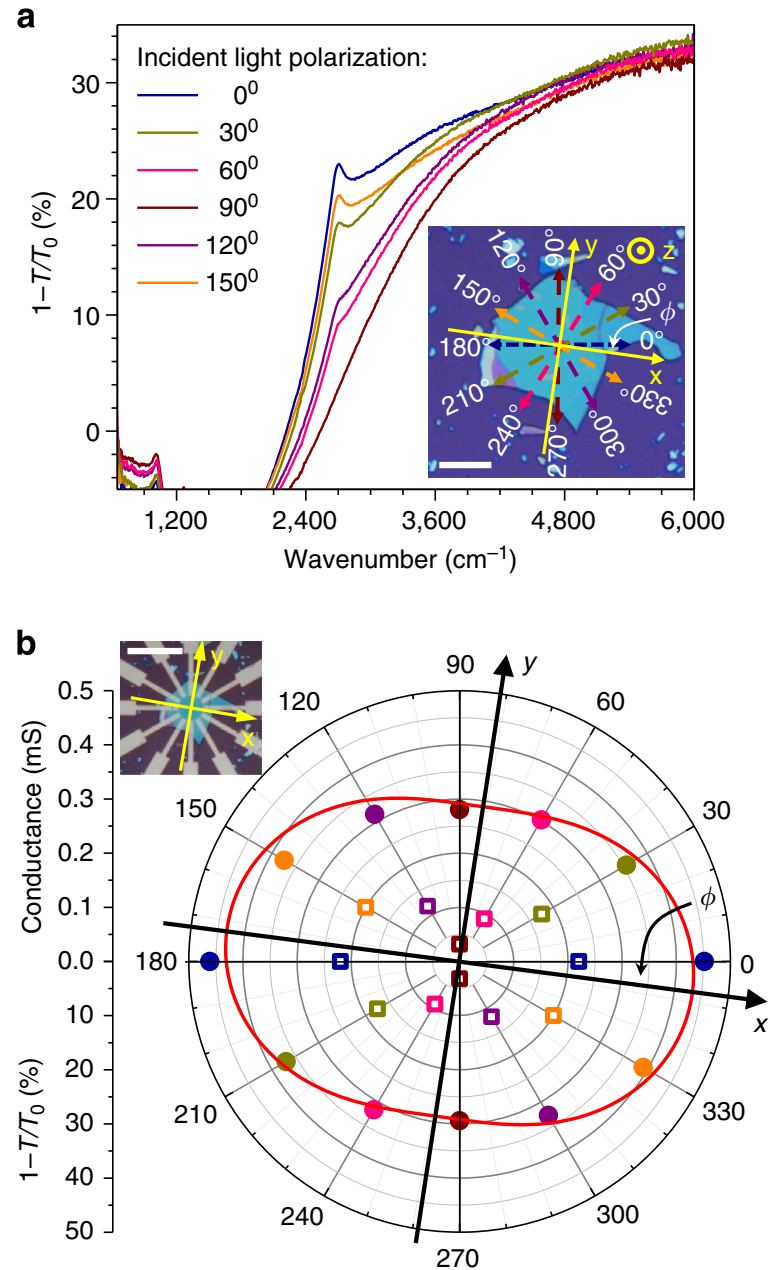

Figure 2 | Polarization-resolved infrared spectroscopy and angleresolved DC conductivity of BP thin film. (a) Polarization-resolved infrared relative extinction spectra when light is polarized along the six directions as shown in the inset. Inset: an optical micrograph of a BP flake with a thickness of around $30 \mathrm{~nm}$. Scale bar: $20 \mu \mathrm{m}$. (b) DC conductivity and $\mathrm{IR}$ relative extinction measured along the same six directions on this $\mathrm{BP}$ flake and plotted in polar coordinates. The angle-resolved DC conductance (solid dots) and the polarization-resolved relative extinction of the same flake at $2,700 \mathrm{~cm}^{-1}$ (hollow squares) are shown on the same polar plot. Dots and squares of the same colour correspond to the directions indicated by the arrows in the inset in $\mathbf{a}$. Inset: an optical image of this BP flake with 12 electrodes spaced at $30^{\circ}$ apart. Scale bar: $50 \mu \mathrm{m}$. The crystalline orientation as determined by polarization-resolved IR measurements and angle-resolved DC conductance measurements match exactly. In both $\mathbf{a}, \mathbf{b}$, $\phi$ is the angle between the $x$ direction and the $0^{\circ}$ reference.

30 degrees, and indicated by the six arrowed lines with different colours in the inset. In this experiment, the $0^{\circ}$ reference direction was selected arbitrarily. For all polarizations, the relative extinction shows a sharp increase at around $2,400 \mathrm{~cm}^{-1}$, indicating a band gap of around $0.3 \mathrm{eV}$, which agrees very well with the previously reported BP band gap at the $z$ symmetry point ${ }^{36}$. Since the optical conductivity of BP peaks in the $x$ direction at the band edge $e^{36}$, by rotating the linear polarizer in finer steps, the angle between the $x$ direction and the blue arrow $\left(0^{\circ}\right.$ in Fig. $\left.2 \mathrm{a}\right)$ is determined to be $-8^{\circ}$. The anisotropy in the optical conductivity arises from the directional dependence of the interband transition matrix elements in anisotropic BP bands ${ }^{36,43}$.
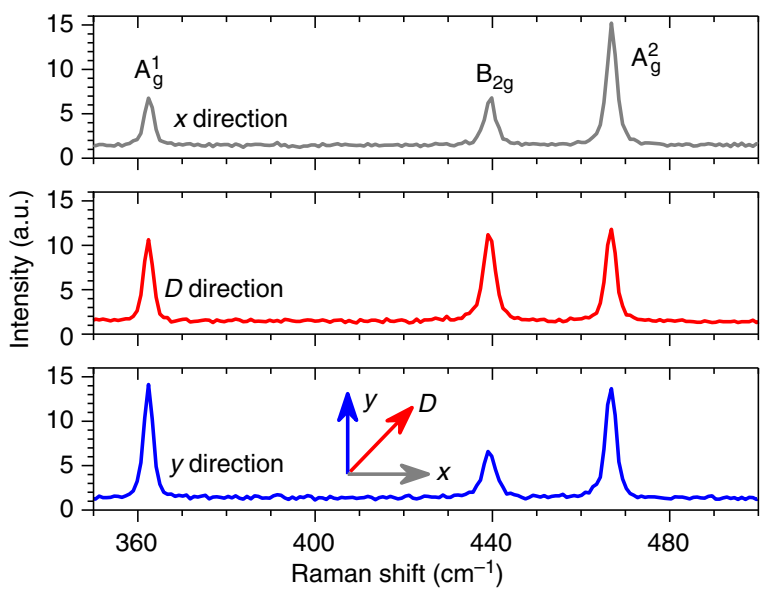

Figure 3 | Polarization-resolved Raman scattering. Polarization-resolved Raman spectra of a BP layered thin film with 532-nm linearly polarized laser excitation incident in the $z$ direction. Raman spectra with excitation laser polarization along $x$ - (grey), $D$ - (red) and $y$ directions (blue) are shown. The $D$ direction is along $45^{\circ}$ angle relative to the $x$ - and $y$ directions, as shown in the bottom panel. All spectra show $A_{g}^{2}, B_{2 g}$ and $A_{g}^{1}$ modes. The peak positions do not change as the excitation light polarization varies. However, the relative intensities of these three modes change significantly with incident light polarization. The polarization-resolved Raman spectra can be used to identify the crystalline orientation of BP thin films, which is especially useful for relatively small flakes due to the small laser spot size $(\sim 1-2 \mu \mathrm{m})$.

Angle-resolved DC conductance. We also independently determined the $x$ - and $y$ directions of the BP thin film using an angleresolved DC conductance measurement. In this experiment, 12 electrodes $(1 \mathrm{~nm} \mathrm{Ti} / 20 \mathrm{~nm} \mathrm{Pd} / 30 \mathrm{~nm} \mathrm{Au})$ were fabricated on the same flake spaced at an angle of $30^{\circ}$ along the directions as shown in the inset of Fig. 2b, which are along the same directions indicated by the coloured lines in Fig. $2 \mathrm{a}$. Here we use the same $0^{\circ}$ reference as that in the polarization-resolved infrared spectroscopy measurement in Fig. 2a. We performed these DC conductance measurements by applying an electric field across each pair of diagonally positioned electrodes separated by $22 \mu \mathrm{m}$ at $180^{\circ}$ apart and the results are plotted in Fig. $2 \mathrm{~b}$ in polar coordinates. Measurements on each pair of electrodes lead to two data points in Fig. $2 \mathrm{~b}$ separated by $180^{\circ}$ for positive and negative bias, respectively. The directional dependence of the low-field conductivity in an anisotropic material can be described by the equation: $\sigma_{\theta}=\sigma_{x} \cos ^{2}(\theta-\phi)+\sigma_{y} \sin ^{2}(\theta-\phi)$. The resulting calculated conductance fits nicely to the measurement data (red solid curve in Fig. 2b). $\sigma_{x}$ and $\sigma_{y}$ refer to the conductivity of BP along the $x$ - and $y$ directions, respectively. $\theta$ is the angle, with respect to the $0^{\circ}$ reference direction, along which both the electric field is applied and the conductance is measured. $\sigma_{\theta}$ is the conductivity along the $\theta$ direction. $\phi$ is the angle between the $x$-direction and the $0^{\circ}$ reference. $\phi=-8^{\circ}$ gives the best fit for this sample (Fig. 2b), which agrees perfectly with the value obtained using infrared spectroscopy. Here, the $x$ - and $y$ directions correspond, respectively, to the high and low mobility directions, as shown in Fig. 1. From the fitted curve, the ratio $\sigma_{x} l$ $\sigma_{y}$ is extracted to be around 1.5 , corresponding to a ratio of the mobility along the $x$ - and $y$ directions $\mu_{x} / \mu_{y}=1.5$. Owing to the current spreading, this simple approach probably leads to an underestimation of the mobility anisotropy, as confirmed by the Hall measurement presented in the later part of this work.

Polarization-resolved Raman scattering. We further performed polarization-resolved Raman scattering measurements on the 


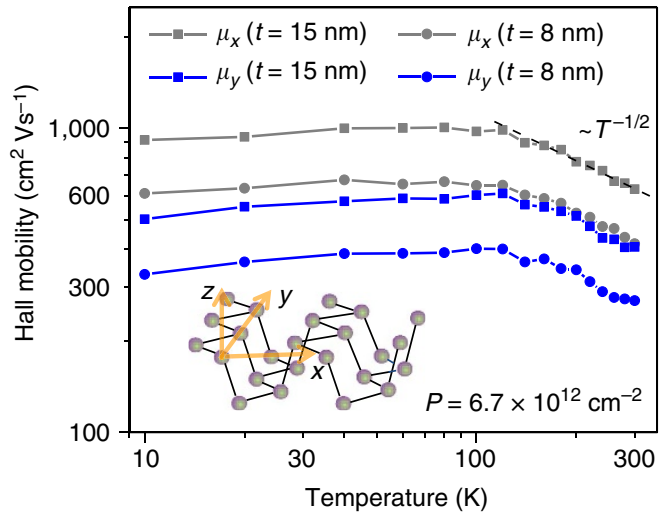

Figure 4 | Angle-resolved Hall mobility. Hall mobilities measured at a constant hole doping concentration of $6.7 \times 10^{12} \mathrm{~cm}^{-2}$ along the $x\left(\mu_{x}\right)$ and $y\left(\mu_{y}\right)$ directions for BP thin films with a thickness of 8 and $15 \mathrm{~nm}$, respectively. $\mu_{x}$ is about 1.8 times as large as $\mu_{y}$ and thicker films consistently show larger mobility than that of thinner films. Inset: schematic view of a single layer BP showing the crystalline directions.

same BP flake discussed in the previous two sections with linearly polarized laser excitation incident from the $z$ direction. This experimental configuration ( $z$ in, $z$ out) is the most relevant for layered BP thin films. The crystalline orientation of this single-crystal flake has already been identified using IR and DC conductivity experiments in the previous sections.

The Raman scattering results for laser excitation light polarizations along the $x, D$ and $y$ directions are plotted in Fig. 3. Here, the $D$ direction is along the $45^{\circ}$ direction to both the $x$ and $y$ directions within the $x-y$ plane. We observe three Raman peaks in thin film BP at around 470, 440 and $365 \mathrm{~cm}^{-1}$, corresponding to the $A_{g}^{2}, B_{2 g}$ and $A_{g}^{1}$ modes, regardless of the polarization, which agrees well with previous observations in bulk BP crystals $^{44}$. These sharp modes correspond to the unique orthorhombic crystalline phosphorus structure, confirming that the material studied in this work is indeed orthorhombic BP. Three other Raman active modes are not observed here because of the selection rules in this particular experimental configuration ( $z$ in, $z$ out). For all three different polarizations, we did not observe measurable peak position variations as the polarization changes. However, the relative intensity of these three peaks does change significantly with polarization direction. For example, as show in Fig. 3, the $B_{2 g}$ mode clearly peaks when incident light is polarized along the $D$-direction because its intensity is determined only by the off-diagonal polarizability tensor $\alpha_{x y}$, which is maximized at $45^{\circ}$ polarization ${ }^{45}$. The relatively intensity of the $\mathrm{A}_{\mathrm{g}}^{1}$ and $\mathrm{A}_{\mathrm{g}}^{2}$ modes varies significantly when the polarization changes. For both $A_{g}$ modes, the Raman scattering intensity is determined by both the diagonal and off-diagonal elements of the polarizability tensor and detailed theoretical investigations are needed to account for these variations. For BP films down to $3 \mathrm{~nm}$, we also observed the same polarization dependence in the relative intensity of the $A_{\mathrm{g}}^{2}, B_{2 g}$ and $A_{g}^{1}$ modes, but again we did not observe any significant frequency shift in these three modes. While a more detailed discussion and modelling of the $(z$ in, $z$ out) polarization-resolved Raman spectroscopy results and their dependence on the thin film thickness is not the focus of this paper, the polarization-resolved Raman measurements presented here offer another method for determining the crystalline directions of the BP thin film crystals.

Angle-resolved Hall mobility. Using the infrared spectroscopy method described above, we first identified the crystalline directions in the BP thin films with a thickness of 8 and $15 \mathrm{~nm}$.
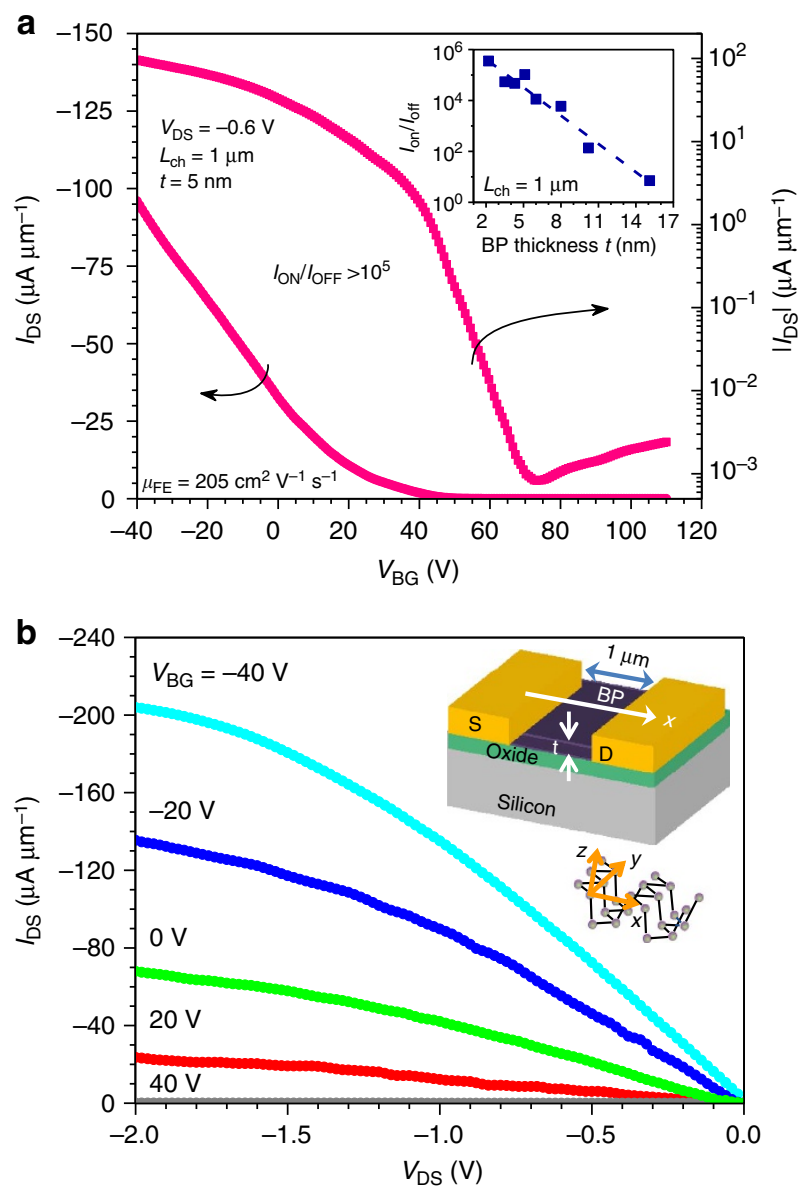

Figure 5 | Black phosphorus field-effect transistors along the $x$ direction. (a) Transfer characteristics of a field-effect transistor with a channel length $L_{\mathrm{ch}}$ of $1 \mu \mathrm{m}$ using a BP thin film of around 5-nm thick. $I_{\mathrm{DS}}$ and $V_{\mathrm{BG}}$ are the source-drain current and back gate bias, respectively. The left and right axes represent linear and logarithmic scales, respectively. Inset: on-off current ratio as a function of BP thickness. (b) Output characteristics of the same transistor showing current saturation. $V_{D S}$ is the drain-source bias. All the transistors have channel lengths of $1 \mu \mathrm{m}$. Inset: a schematic view of the transistor. The $x$-direction is indicated by the arrow. The black phosphorus crystalline directions $(x, y$, and $z)$ are shown below the transistor schematic.

Standard Hall bars were fabricated on these thin films and the mobilities were measured in both the $x$ - and $y$ directions from $10 \mathrm{~K}$ to room temperature, as shown in Fig. 4 . For the $15 \mathrm{~nm}$ sample, the mobility along the $x$ direction exceeds $600 \mathrm{~cm}^{2} \mathrm{~V}^{-1}$ $\mathrm{s}^{-1}$ at room temperature and is above $1,000 \mathrm{~cm}^{2} \mathrm{~V}^{-1} \mathrm{~s}^{-1}$ at $120 \mathrm{~K}$. For the $8 \mathrm{~nm}$ sample, the mobility along the $x$ direction exceeds $400 \mathrm{~cm}^{2} \mathrm{~V}^{-1} \mathrm{~s}^{-1}$ at room temperature and is above $600 \mathrm{~cm}^{2} \mathrm{~V}^{-1} \mathrm{~s}^{-1}$ at $120 \mathrm{~K}$. The hole carrier density is kept at $6.7 \times 10^{12} \mathrm{~cm}^{-2}$ in all the measurements above. For both thicknesses, the Hall mobility along the $x$ direction is about 1.8 times as large as that in the $y$ direction, representing an anisotropy slightly smaller than that in bulk $\mathrm{BP}^{36}$ but slightly larger than that extracted from the DC conductance measurement in Fig. $2 \mathrm{~b}$. Thicker BP consistently shows a higher mobility, indicating the impact of the substrate on the scattering of the carriers in BP thin films ${ }^{46}$. In bulk BP crystals, hole mobility values above 1,100 and $50,000 \mathrm{~cm}^{2} \mathrm{~V}^{-1} \mathrm{~s}^{-1}$ along the $x$ direction were previously reported at 300 and $30 \mathrm{~K}$, respectively ${ }^{36}$. As a result, future improvement in BP thin film material quality is likely to result in much enhanced mobilities. The temperature dependence of the 
carrier mobility in BP shows a characteristic behaviour found in other layered materials. The initial increase in carrier mobility from 10 to $100 \mathrm{~K}$ is most likely due to the dominating Coulomb scattering mechanism ${ }^{47,48}$. As the temperature further increases, the mobility decreases due to phonon scattering following the power law relation $\sim T^{-\gamma}$, with $\gamma$ close to 0.5 . This power law dependence is apparently weaker than in other $2 \mathrm{D}$ materials such as graphene and $\mathrm{TMDCs}^{49}$. Further theoretical and experimental lines of work are needed to clarify the temperature dependence of the mobility.

Transistors using $x$ direction BP thin film as a channel material. We also fabricated a series of transistors with a channel length of $1 \mu \mathrm{m}$ using BP flakes with thicknesses varying from 2 to $15 \mathrm{~nm}$. The transistor channels were intentionally designed along the $x$ direction, as shown in the inset of Fig. 5b, which was determined using either infrared spectroscopy or Raman scattering as discussed above. Figure $5 \mathrm{a}, \mathrm{b}$ denotes the transfer and output characteristics of a BP transistor at room temperature with a thickness of around $5 \mathrm{~nm}$. An on-off current ratio exceeding $10^{5}$ is achieved. In addition, plotted in Fig. $5 \mathrm{a}$ are the transfer characteristics on a linear scale, from which a field-effect mobility of $205 \mathrm{~cm}^{2} \mathrm{~V}^{-1} \mathrm{~s}^{-1}$ is inferred using $\mu_{\mathrm{FE}}=\frac{\mathrm{d} I_{\mathrm{DS}}}{\mathrm{d} V_{\mathrm{BG}}} \frac{L}{C_{\mathrm{ox}} W V_{\mathrm{DS}}}$, where $I_{\mathrm{DS}}$ is the source-drain current, $V_{\mathrm{BG}}$ is the back gate bias, $V_{\mathrm{DS}}$ is the source-drain bias, $C_{\mathrm{ox}}$ is the gate capacitance, $W$ is the width of the transistor and $L$ is the channel length. Here, we emphasize that the field-effect mobility may be strongly affected by the traps in the device and the intrinsic band mobility can be much higher than $205 \mathrm{~cm}^{2} \mathrm{~V}^{-1} \mathrm{~s}^{-1}$, as shown in $\mathrm{MoS}_{2}$ transistors ${ }^{50}$. Compared with graphene transistors, the BP devices consistently show much improved current saturation behaviour as shown in Fig. 5b. Finally, we plot the scaling of the on-off current ratio as a function of the BP thickness in the inset of Fig. 5a. For a BP thickness of $8 \mathrm{~nm}$, the transistor on-off current ratio is $10^{4}$ and the field-effect mobility is $300 \mathrm{~cm}^{2} \mathrm{~V}^{-1} \mathrm{~s}^{-1}$. For a BP thickness of around $2 \mathrm{~nm}$, the on-off current ratio is as high as $5 \times 10^{5}$. The field-effect mobility of this BP transistor is around $50 \mathrm{~cm}^{2} \mathrm{~V}^{-1} \mathrm{~s}^{-1}$, probably due to the greatly enhanced scattering by the substrate. However, we want to emphasize that this field-effect mobility may not represent the true carrier mobility because of traps and contact resistance ${ }^{50}$. The much improved transistor performance as compared with graphene indicates that the BP transistors can overcome the major shortcomings of graphene transistors in terms of on-off current ratio and current saturation. Combined with a much higher mobility than TMDCs, BP devices have great potential for applications in thin film radio-frequency electronics and logic circuits. Finally, as shown in Fig. $5 \mathrm{a}$, the charge neutrality is achieved at a gate bias of around $70 \mathrm{~V}$, corresponding to a hole doping of around $5 \times 10^{12} \mathrm{~cm}^{-2}$. The exact origin of the doping is not clear and can be due to the adsorbates and contamination, and they may also adversely affect the carrier mobility. The intrinsic carrier mobility and doping mechanisms are important future research topics.

\section{Discussions}

In summary, we have reintroduced BP as an anisotropic material with a narrow bandgap and a high carrier mobility to the layered-material family. It bridges the energy gap between the semimetallic graphene and various large bandgap TMDCs, making BP thin films an ideal candidate for various near and mid-infrared optoelectronic applications, such as photodetectors and modulators. Moreover, because of its relatively high carrier mobility and good current saturation properties, thin-film BP has great potential to surpass graphene as a novel and more favourable material for high performance $\mathrm{RF}$ and logic applications in thin-film electronics. Most importantly, its unique angle-dependent properties allow a new degree of freedom for designing conceptually new optoelectronic and electronic devices, which are not possible using other 2D materials. For example, BP is a natural material for developing mid-infrared polarizers and polarization sensors, while its angle-dependent optical conductivity may allow the construction of plasmonic devices where the surface plasmon polariton frequency will have a strong directional dependence on the wave vector. Owing to its anisotropic nature, BP also offers advantages for building better transistors along its light effective-mass $(x)$ direction. The low effective mass can help to obtain high-performance quasi-ballistic transistors at advanced technology nodes where the channel lengths are comparable to the mean free path of carriers by achieving higher low-field mobility, overshoot velocity and onstate current ${ }^{25-27}$. These are just a few of the promising applications of BP that we have identified, while an entire family of novel device concepts that can utilize the angledependent properties of this intriguing material awaits our exploration.

During the preparation of this manuscript, we became aware of two experimental works studying the electronic properties of BP on arXiv.org (arXiv:1401.4117v1 and arXiv:1401.4133v1). During the revision process, these two manuscripts and one additional experimental work were published ${ }^{51-53}$.

\section{Methods}

Transistor and Hall bar fabrication. The fabrication of our devices starts with the exfoliation of BP thin films from bulk BP crystals on $300 \mathrm{~nm} \mathrm{SiO}{ }_{2}$ on a Si substrate which has pre-patterned alignment grids, using the micromechanical cleavage technique. The thickness of the $\mathrm{SiO}_{2}$ was selected to provide the optimal optical contrast for locating BP flakes relative to the alignment grids and for roughly estimating their thicknesses. The number of BP layers was then confirmed using AFM based on the sample thickness. The next step was to pattern the resist layer for metallization using a Vistec $100 \mathrm{kV}$ electron-beam lithography system based on poly (methyl methacrylate) (950k MW PMMA). PMMA A3 was spun on wafer at a speed of 3,000 r.p.m. and was then bakes at $175^{\circ}$ for $3 \mathrm{~min}$. The dose for exposure is $1,100 \mu \mathrm{Ccm}^{-2}$. Development was performed in 1:3 MIBK: IPA (Methyl isobutyl ketone: Isopropanol). We then evaporated $1 \mathrm{~nm} \mathrm{Ti} / 20 \mathrm{~nm} \mathrm{Pd} / 30 \mathrm{~nm}$ Au followed by lift-off in acetone to form the contacts.

AFM and Raman spectroscopy. AFM for identifying the thin-film thickness was performed on a Digital Instruments/Veeco Dimension 3000 system. Polarizationresolved Raman spectroscopy was performed using a 532-nm Nd:YAG laser in the LabRAM ARAMIS system. We used a $\times 100$ microscope objective and the power incident on BP was kept below $500 \mu \mathrm{W}$ to avoid sample damage.

Infrared spectroscopy. Infrared spectroscopy was performed in the 800$6,000 \mathrm{~cm}^{-1}$ range using a Bruker Optics Fourier Transfer Infrared spectrometer (Vertex 70) integrated with a Hyperion 2000 microscope system. Incident light polarization was achieved using an infrared polarizer.

Electrical characterization. All electrical characterizations were performed using an Agilent 4155C semiconductor parameter analyser and a Lakeshore cryogenic probe station with micromanipulation probes.

\section{References}

1. Novoselov, K. S. et al. Electric field effect in atomically thin carbon films. Science 306, 666-669 (2004)

2. Novoselov, K. S. et al. Two-dimensional gas of massless Dirac fermions in graphene. Nature 438, 197-200 (2005).

3. Zhang, Y., Tan, Y. W., Stormer, H. L. \& Kim, P. Experimental observation of the quantum Hall effect and Berry's phase in graphene. Nature 438, 201-204 (2005).

4. Nair, R. R. et al. Fine structure constant defines visual transparency of graphene. Science 320, 1308 (2008).

5. Mak, K. F. et al. Measurement of the optical conductivity of graphene. Phys. Rev. Lett. 101, 196405 (2008).

6. Lin, Y. M. et al. $100-\mathrm{GHz}$ transistors from wafer-Scale epitaxial graphene. Science 327, 662 (2010).

7. Lee, C., Wei, X., Kysar, J. W. \& Hone, J. Measurement of the elastic properties and intrinsic strength of monolayer graphene. Science 321, 385-388 (2008) 
8. Bonaccorso, F., Sun, Z., Hasan, T. \& Ferrari, A. C. Graphene photonics and optoelectronics. Nat. Photon. 4, 611-622 (2010).

9. Jia, X. et al. Controlled formation of sharp zigzag and armchair edges in graphitic nanoribbons. Science 323, 1701-1705 (2009).

10. Schwierz, F. Graphene transistors. Nat. Nanotechnol. 5, 487-496 (2010).

11. Dean, C. R. et al. Boron nitride substrates for high-quality graphene electronics. Nat. Nantechnol. 5, 722-726 (2010).

12. Alem, S. et al. Atomically thin hexagonal boron nitride probed by ultrahigh-resolution transmission electron microscopy. Phys. Rev. B 80, 155425 (2009).

13. Xue, J. et al. Scanning tunnelling microscopy and spectroscopy of ultra-flat graphene on hexagonal boron nitride. Nat. Mater. 10, 282-285 (2011).

14. Novoselov, K. S. et al. Two-dimensional atomic crystals. Proc. Natl Acad. Sci. USA 102, 10451-10453 (2005).

15. Radisavljevic, B. et al. Single-layer $\mathrm{MoS}_{2}$ transistors. Nat. Nanotechnol. 6, 147-150 (2011).

16. Mak, K. F. et al. Atomically thin $\mathrm{MoS}_{2}$ : a new direct-gap semiconductor. Phys. Rev. Lett. 105, 136805 (2010).

17. Splendiani, A. et al. Emerging photoluminescence in monolayer $\mathrm{MoS}_{2}$. Nano Lett. 10, 1271-1275 (2010).

18. Jones, A. M. et al. Spin-layer locking effects in optical orientation of exciton spin in bilayer WSe $\mathrm{W}_{2}$. Nat. Phys. 10, 130-134 (2014).

19. Britnell, L. et al. Strong light-matter interactions in heterostructures of atomically thin films. Science 340, 1311-1314 (2013).

20. Chhowalla, M. et al. The chemistry of two-dimensional layered transition metal dichalcogenide nanosheets. Nat. Chem. 5, 263-275 (2013).

21. Fang, H. et al. High-performance single layered WSe $\mathrm{W}_{2} \mathrm{p}-\mathrm{FET}$ s with chemically doped contacts. Nano Lett. 12, 3788-3792 (2012).

22. Wang, H. et al. Integrated circuits based on bilayer $\mathrm{MoS}_{2}$ transistors. Nano Lett. 12, 4674-4680 (2012)

23. Fuhrer, M. S. \& Hone, J. Measurement of mobility in dual-gated $\mathrm{MoS}_{2}$ transistors. Nat. Nanotechnol. 8, 146-147 (2013).

24. Low, T. et al.Plasmons and screening in monolayer and multilayer black phosphorus. Preprint at http://arxiv.org/abs/1404.4035 (2014).

25. Abudukelimu, A. et al. The effect of isotropic and anisotropic scattering in drain region of ballistic channel diode. IEEE Int. Conf. Solid State Integr. Circuit Technol. 1247-1249 (2010).

26. Bufler, F. \& Fichtner, W. Scaling of strained-Si n-MOSFETs into the ballistic regime and associated anisotropic effects. IEEE Trans. Electron Devices 50, 278-284 (2003)

27. Bufler, F., Keith, S. \& Meinerzhagen, B. Anisotropic ballistic in-plane transport of electrons in strained Si. Proc. SISPAD 239-242 (1998).

28. Keyes, R. The electrical properties of black phosphorus. Phys. Rev. 92, 580-584 (1953).

29. Warschauer, D. Electrical and optical properties of crystalline black phosphorus. J. Appl. Phys. 34, 1853-1860 (1963).

30. Jamieson, J. Crystal structures adopted by black phosphorus at high pressures. Science 139, 1291-1292 (1963).

31. Wittig, J. \& Matthias, B. T. Superconducting phosphorus. Science 160, 994-995 (1968).

32. Maruyama, Y., Suzuki, S., Kobayashi, K. \& Tanuma, S. Synthesis and some properties of black phosphorus single crystals. Physica 105B, 99-102 (1981).

33. Sugai, S. Raman and infrared reflection spectroscopy in black phosphorus. Solid State Commun. 53, 753-755 (1985).

34. Asahina, H. \& Morita, A. Band structure and optical properties of black phosphorus. J. Phys. C Solid State Phys. 17, 1839-1852 (1984).

35. Takahashi, T. et al. Electronic band structure of black phosphorus studied by angle-resolved ultraviolet photoelectron spectroscopy. J. Phys. C Solid State Phys. 18, 825-836 (1985).

36. Morita, A. Semiconducting black phosphorus. Appl. Phys. A 39, 227-242 (1986).

37. Du, Y., Ouyang, C., Shi, S. \& Lei, M. Ab initio studies on atomic and electronic structures of black phosphorus. J. Appl. Phys. 107, 093718 (2010).

38. Tran, V., Soklaski, R., Liang, Y. \& Yang, L. Tunable band gap and anisotropic optical response in few-layer black phosphorus. Preprint at http://arxiv.org/abs/ 1402.4192 (2014).
39. Qiao, J., Kong, X., Hu, Z., Yang, F. \& Ji, W. Few-layer black phosphorus: emerging 2D semiconductor with high anisotropic carrier mobility and linear dichroism. Preprint at http://arxiv.org/abs/1401.5045 (2014).

40. Rodin, A. S., Carvalho, A. \& Castro Neto, A. H. Strain-induced gap modification in black phosphorus. Phys. Rev. Lett. 112, 176801 (2014).

41. Yan, H. et al. Tunable infrared plasmonic devices using graphene/insulator stacks. Nat. Nanotechnol. 7, 330-334 (2012).

42. Yan, H. et al. Damping pathways of mid-infrared plasmons in graphene nanostructures. Nat. Photon. 7, 394-399 (2013).

43. Asahina, H., Shindo, K. \& Morita, A. Electronic structure of black phosphorus in self-consistent pseudopotential approach. J. Phys. Soc. Jpn 51, 1193-1199 (1982).

44. Akahama, Y., Kobayashi, M. \& Kawamura, H. Raman study of black phosphorus up to 13GPa. Solid State Commun. 104, 311-315 (1997).

45. Lazzeri, M. \& Mauri, F. First-principles calculation of vibrational Raman spectra in large systems: signature of small rings in crystalline $\mathrm{SiO}_{2}$. Phys. Rev. Lett. 90, 036401 (2003).

46. Perebeinos, V., Rotkin, S., Petrov, A. G. \& Avouris, P. The effects of substrate phonon mode scattering on transport in carbon nanotubes. Nano Lett. $\mathbf{9}$, 312-316 (2009)

47. Chen, J. H. et al. Charged-impurity scattering in graphene. Nat. Phys. 4, 377-381 (2008).

48. Zhu, W., Perebeinos, V., Freitag, M. \& Avouris, P. Carrier scattering, mobilities, and electrostatic potential in monolayer, bilayer, and trilayer graphene. Phys. Rev. B 80, 235402 (2009).

49. Radisavljevic, B. \& Kis, A. Mobility engineering and a metal-insulator transition in monolayer $\mathrm{MoS}_{2}$. Nat. Mater. 12, 815-820 (2013).

50. Zhu, W. et al. Electronic transport and device prospects of monolayer molybdenum disulphide grown by chemical vapour deposition. Nat. Commun. 5, 3087 (2014).

51. Li, L. et al. Black phosphorus field-effect transistors. Nat. Nanotechnol. $\mathbf{9}$, 372-377 (2014).

52. Liu, H. et al. Phosphorene: an unexplored $2 \mathrm{D}$ semiconductor with a high hole mobility. ACS Nano 8, 4033-4041 (2014).

53. Koenig, S., Doganov, R., Schmidt, H., Castro Neto, A. \& Ozyilmaz, B. Electric field effect in ultrathin black phosphorus. Appl. Phys. Lett. 104, 103106 (2014)

\section{Acknowledgements}

We thank M. Dresselhaus at MIT for discussions on crystal anisotropy in layered materials and detailed comments on the manuscript. We thank T.-P. Ma at Yale University and S. Rakheja at MIT for stimulating discussions on the ballistic transport and transistor performance. We would also like to thank T. Low at Yale University and IBM for discussions on the Raman measurements, optical and DC conductivity anisotropy in BP. The authors also thank M. Dubey at Army Research Laboratory (ARL) for insightful comments on the manuscript. We are grateful to S. Guha, W. Haensch, P. Avouris, S. Assefa and S.-J. Han at IBM for their support of this work. We thank W. Zhu, B. Ek, Q. Cao and J. Bucchignano at IBM, and M. Rooks at Yale University for technical assistance. F.X. acknowledges partial financial support from the Office of Naval Research (ONR).

\section{Author contributions}

F.X. and H.W. conceived the original ideas of this study, designed and performed the experiments, and co-wrote the manuscript. Y.J. helped with sample preparation, device fabrication and equipment set-up. All the authors discussed the results and commented on the manuscript.

\section{Additional information}

Competing financial interests: The authors declare no competing financial interests.

Reprints and permission information is available online at http://npg.nature.com/ reprintsandpermissions/

How to cite this article: Xia, F. et al. Rediscovering black phosphorus as an anisotropic layered material for optoelectronics and electronics. Nat. Commun. 5:4458 doi: $10.1038 /$ ncomms5458 (2014). 